\title{
Equation of state for directed percolation
}

\author{
H K Janssen, Ü Kutbay and K Oerding \\ Institut für Theoretische Physik III, \\ Heinrich-Heine-Universität, 40225 Düsseldorf, Germany
}

\begin{abstract}
Using field-theoretic renormalization group methods we calculate the equation of state for non-equilibrium systems belonging to the universality class of directed percolation (Gribov process) to second order in $\epsilon=4-d$. By introducing a parametric representation the result can be written to this order in a very simple form. We use our result to obtain a universal amplitude ratio to second order in $\epsilon$.
\end{abstract}

PACS numbers: 05.40.+j, 64.60.Ak, 64.60.Ht

Short title: Equation of state for directed percolation

November 12, 2017 


\section{Introduction}

Numerous physical systems that show continuous non-equilibrium phase transitions display near the transition point space-time structures that can be described by directed percolation clusters. Well-known examples are epidemic processes without immunization [1], poisoning of catalytic surfaces [2], roughening transitions in growth processes [3], Schlögl's reaction models [4, 5], contact processes [6, 7] and certain cellular automata 8, 9. The model known in particle physics as reggeon field theory [10, 11, 12] has also been related to directed percolation and epidemic processes [13, 14, 15, 16]. All these systems share the property that their critical behaviour is characterized by the existence of an absorbing state. This property determines their universality class [5, 17].

The behaviour of these systems near the transition point is characterized by universal quantities such as critical exponents. The exponents for directed percolation have been calculated to second order in $\epsilon=4-d$ [5] and-numerically-for $d=1$ and $d=2$ (for recent results see, e.g., [18, 19, 20]). It is well-known from the theory of equilibrium critical phenomena that universality not only holds for critical exponents but also for certain amplitude ratios and scaling functions [21, 22, 23]. An example for a universal relationship between physical quantities near a critical point is the equation of state. In the present paper we derive an equation that describes the stationary state of epidemic processes with an absorbing state near the transition point.

Before we turn to the mesoscopic description used in the analysis presented below let us briefly discuss two examples for microscopic models that belong to the universality class of directed percolation. Schlögl's autocatalytic reaction scheme «1 is defined by the reaction equations

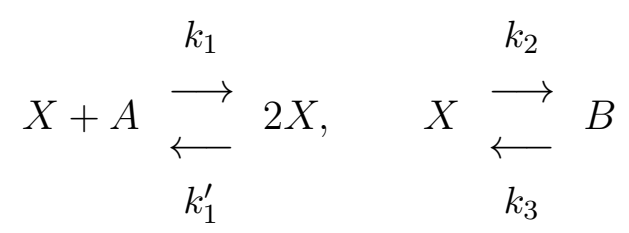

where the concentration of $A$ 's and $B$ 's is kept constant (e.g., by reservoirs). Here two remarks are in order: (i) If $A=B$ (i.e., the particles are of the same type) and their concentration is not kept constant (but governed by the reaction-diffusion dynamics) the system belongs to a different universality class. In that case the total particle density is conserved and affects the long-time dynamics of the system [24, 25]. (ii) Only if the reaction $B \rightarrow X$ has zero probability the system has an absorbing state. In the case of chemical systems it is natural to take this process into account since back reactions can in general not be avoided. Thus the B-particles represent a source for X. Below a critical value of the reaction rate $k_{2}$ the density of $X$-particles is nonvanishing in the stationary state for $k_{3} \rightarrow 0$.

In the theory of infectious diseases [1] one considers two types of individuals: 
Susceptibles S who can catch the disease and infectives I who have the disease and can transmit it. The dynamics of the system is characterized by the following processes:

(i) Healthy (susceptible) individuals may catch the disease by contact with infectives. Infected individuals may recover spontaneously and thereby become susceptible again.

(ii) Interaction of infected individuals can lead to recovery ("saturation").

(iii) Diffusive spreading of the disease.

(iv) Susceptibles may catch the disease spontaneously.

The process (iv) models for instance a continuos flow of germs into the system. Note that the system has an absorbing state if (and only if) the process (iv) has zero probability.

A mesoscopic description for the dynamics near the transition point requires a single density $n(\mathbf{r}, t)$ which represents the slow degrees of freedom. In the case of Schlögl's model (11) $n$ corresponds to the density of $X$-particles, in the context of poisoning of a catalytic surface $n$ is the density of vacant sites and in epidemic processes it represents the density of infectives. The density satisfies the Langevin equation

$$
\partial_{t} n=\lambda \nabla^{2} n+R[n] n+\lambda q+\zeta
$$

where the reaction rate $R[n]$ can be expanded in powers of $n$. In order to investigate the universal properties near the transition point we only need the two leading terms,

$$
R[n]=\lambda\left(\tau+\frac{g}{2} n\right)
$$

where $g>0$ and $\tau$ is a temperature-like critical parameter. For $q=0$ the system has an absorbing state with $n=0$ if the random force $\zeta$ is multiplicative:

$$
\left\langle\zeta(\mathbf{r}, t) \zeta\left(\mathbf{r}^{\prime}, t^{\prime}\right)\right\rangle=\lambda \tilde{g} n(\mathbf{r}, t) \delta\left(\mathbf{r}-\mathbf{r}^{\prime}\right) \delta\left(t-t^{\prime}\right)
$$

where we have again neglected irrelevant higher powers in $n$. In the case of the reactiondiffusion system (1) the (constant) source term $q$ vanishes for a zero reaction rate $k_{3}$. By a simple rescaling it is possible to render the coupling constants $g$ and $\tilde{g}$ equal.

\section{Renormalized field theory and perturbational calculation of the equation of state}

Hereafter we work with the dynamic functional [5, 29, 30, 31, 32]

$$
\mathcal{J}[\tilde{s}, s]=\int \mathrm{d} t \int \mathrm{d}^{d} r \tilde{s}\left[\partial_{t} s+\lambda\left(\tau-\nabla^{2}\right) s+\frac{\lambda g}{2} s(s-\tilde{s})-\lambda q\right]
$$

where $s \propto n$ and $\tilde{s}$ denotes a Martin-Siggia-Rose response field [33]. Response and correlation functions can be computed by integrating products of the fields $\tilde{s}$ and 
$s$ with the weight $\exp (-\mathcal{J})$. We are especially interested in the average $M=\langle s\rangle$ which corresponds to the mean particle density. The external field $\lambda q$ describes the spontaneous creation of particles and breaks the symmetry $\tilde{s}(t) \leftrightarrow-s(-t)$.

In order to obtain the equation of state -i.e., $M$ as a function of $\tau$ and $q$ in the stationary state - we perform the shift $s=M+\phi$ und determine $M$ by the no-tadpole requirement $\langle\phi\rangle=0$. Since the stationary state is homogeneous the shift leads to the functional

$$
\mathcal{J}_{\phi}[\tilde{\phi}, \phi]=\mathcal{J}_{0}[\tilde{\phi}, \phi]+\mathcal{J}_{G}[\tilde{\phi}, \phi]+\mathcal{J}_{I}[\tilde{\phi}, \phi]
$$

where $\tilde{\phi}=\tilde{s}$,

$$
\begin{aligned}
& \mathcal{J}_{0}[\tilde{\phi}, \phi]=\int \mathrm{d} t \int \mathrm{d}^{d} r \lambda \tilde{\phi}\left[M\left(\tau M+\frac{g}{2} M\right)-q\right] \\
& \mathcal{J}_{G}[\tilde{\phi}, \phi]=\int \mathrm{d} t \int \mathrm{d}^{d} r \tilde{\phi}\left[\partial_{t} \phi+\lambda\left(\tau+g M-\nabla^{2}\right) \phi-\frac{\lambda g}{2} M \tilde{\phi}\right]
\end{aligned}
$$

and

$$
\mathcal{J}_{I}=\int \mathrm{d} t \int \mathrm{d}^{d} r \frac{\lambda g}{2} \tilde{\phi} \phi(\phi-\tilde{\phi})
$$

The equation of state can now be written in the form

$$
\lambda q=\lambda M\left(\tau+\frac{g}{2} M\right)-\leftarrow
$$

where the bubble represents the sum of all one-particle irreducible Feynman diagrams of the field theory (6)-(9) with one external $\tilde{\phi}$-leg. Details of the diagrammatic analysis are given in the appendix. After dimensional regularization of ultraviolet divergences one obtains at two-loop order

$q=M\left[\tau+\frac{g}{2} M-\frac{G_{\epsilon} g^{2} \bar{\tau}^{1-\epsilon / 2}}{\epsilon(2-\epsilon)}-\frac{g^{4} \bar{\tau}^{-\epsilon}}{24}\left(g M \frac{1-\epsilon}{2} I_{1}+\left(2 I_{1}+3 I_{2}\right) \bar{\tau}\right)\right]$

where $G_{\epsilon}=\Gamma(1+\epsilon / 2) /(4 \pi)^{d / 2}$ is a geometrical factor and

$$
\bar{\tau}=\tau+g M
$$

The integrals $I_{1}$ and $I_{2}$ are defined in the appendix.

The poles in $\epsilon$ can be absorbed into renormalizations of the fields and the coupling constants. The renormalizations read

$$
\begin{aligned}
& \tilde{\phi} \rightarrow \stackrel{\circ}{\phi}=Z^{1 / 2} \tilde{\phi} \quad \phi \rightarrow \stackrel{\circ}{\phi}=Z^{1 / 2} \phi \quad M \rightarrow \stackrel{\circ}{M}=Z^{1 / 2} M \\
& q \rightarrow \stackrel{\circ}{q}=Z_{\lambda}^{-1} Z^{1 / 2} q \quad \lambda \rightarrow \stackrel{\circ}{\lambda}=Z^{-1} Z_{\lambda} \lambda \quad \tau \rightarrow \stackrel{\circ}{\tau}=Z_{\lambda}^{-1} Z_{\tau} \tau \\
& g \rightarrow \stackrel{\circ}{g}=Z^{-1 / 2} Z_{\lambda}^{-1} Z_{g} g \quad G_{\epsilon} g^{2}=u \mu^{\epsilon}
\end{aligned}
$$


where $\mu$ denotes an external momentum scale. The required renormalization factors have been determined to two loop order in Ref. [5]. The Z-factors are

$$
\begin{aligned}
& Z=1+\frac{u}{4 \epsilon}+\left(\frac{7}{\epsilon}-3+\frac{9}{2} \ln \frac{4}{3}\right) \frac{u^{2}}{32 \epsilon} \\
& Z_{\lambda}=1+\frac{u}{8 \epsilon}+\left(\frac{13}{\epsilon}-\frac{31}{4}+\frac{35}{2} \ln \frac{4}{3}\right) \frac{u^{2}}{128 \epsilon} \\
& Z_{\tau}=1+\frac{u}{2 \epsilon}+\left(\frac{1}{2 \epsilon^{2}}-\frac{5}{32 \epsilon}\right) u^{2} \quad Z_{g}=1+\frac{u}{\epsilon}+\left(\frac{5}{4 \epsilon^{2}}-\frac{7}{16 \epsilon}\right) u^{2} .
\end{aligned}
$$

\section{Scaling form of the equation of state}

In order to improve the perturbative result (11) by the renormalization group we exploit as usual the invariance of the bare field theory with respect the a variation of $\mu$ at fixed bare parameters, i.e.,

$$
\left.\mu \frac{\mathrm{d}}{\mathrm{d} \mu}\right|_{0} \stackrel{q}{q}(\stackrel{\circ}{\tau}, \stackrel{\circ}{M}, \stackrel{\circ}{g})=0 .
$$

This equation - expressed by renormalized quantities - is the renormalization group equation

$$
\left[\mu \partial_{\mu}+\kappa \tau \partial_{\tau}-\frac{1}{2} \gamma M \partial_{M}+\beta \partial_{u}+\zeta-\frac{1}{2} \gamma\right] q(\tau, M, u ; \mu)=0
$$

with the Wilson functions (to two-loop order)

$$
\begin{aligned}
& \gamma(u)=\left.\frac{\mathrm{d} \ln Z}{\mathrm{~d} \ln \mu}\right|_{0}=-\frac{u}{4}+\left(3-\frac{9}{2} \ln \frac{4}{3}\right) \frac{u^{2}}{16} \\
& \kappa(u)=\left.\frac{\mathrm{d} \ln \tau}{\mathrm{d} \ln \mu}\right|_{0}=\frac{3 u}{8}-\left(\frac{49}{4}+\frac{35}{2} \ln \frac{4}{3}\right) \frac{u^{2}}{64} \\
& \zeta(u)=\left.\frac{\mathrm{d} \ln \lambda}{\mathrm{d} \ln \mu}\right|_{0}=-\frac{u}{8}+\left(\frac{17}{2}-\ln \frac{4}{3}\right) \frac{u^{2}}{128} \\
& \beta(u)=\left.\frac{\mathrm{d} u}{\mathrm{~d} \ln \mu}\right|_{0}=u\left[-\epsilon+\frac{3 u}{2}-\left(169+106 \ln \frac{4}{3}\right) \frac{u^{2}}{128}\right] .
\end{aligned}
$$

The renormalization group equation (16) can be solved by characteristics. At the fixed point $\left(u_{\star}\right.$ with $\left.\beta\left(u_{\star}\right)=0\right)$

$$
u_{\star}=\frac{2 \epsilon}{3}\left[1+\left(\frac{169}{192}+\frac{53}{96} \ln \frac{4}{3}\right) \frac{2 \epsilon}{3}+\mathrm{O}\left(\epsilon^{2}\right)\right]
$$

the result (combined with dimensional analysis) reads

$$
q(\tau, M, u ; \mu)=\mu^{2+d / 2} l^{z+(d-\eta) / 2} q\left(\mu^{-2} l^{-1 / \nu} \tau, \mu^{-d / 2} l^{-(d+\eta) / 2} M, u_{\star} ; 1\right)
$$


with the critical exponents [5]

$$
\begin{aligned}
& \eta=\gamma\left(u_{\star}\right)=-\frac{\epsilon}{6}\left[1+\left(\frac{25}{288}+\frac{161}{144} \ln \frac{4}{3}\right) \epsilon+\mathrm{O}\left(\epsilon^{2}\right)\right] \\
& \nu=\frac{1}{2-\kappa\left(u_{\star}\right)}=\frac{1}{2}+\frac{\epsilon}{16}\left[1+\left(\frac{107}{288}-\frac{17}{144} \ln \frac{4}{3}\right) \epsilon+\mathrm{O}\left(\epsilon^{2}\right)\right] \\
& z=2+\zeta\left(u_{\star}\right)=2-\frac{\epsilon}{12}\left[1+\left(\frac{67}{288}+\frac{59}{144} \ln \frac{4}{3}\right) \epsilon+\mathrm{O}\left(\epsilon^{2}\right)\right] \\
& \beta=\frac{\nu(d+\eta)}{2}=1-\frac{\epsilon}{6}\left[1-\left(\frac{11}{288}-\frac{53}{144} \ln \frac{4}{3}\right) \epsilon+\mathrm{O}\left(\epsilon^{2}\right)\right] \\
& \delta=1+\frac{\nu(z-\eta)}{\beta}=2+\frac{\epsilon}{3}\left[1+\left(\frac{85}{288}+\frac{53}{144} \ln \frac{4}{3}\right) \epsilon+\mathrm{O}\left(\epsilon^{2}\right)\right] .
\end{aligned}
$$

and the scaling function

$$
\begin{aligned}
q\left(\tau, M, u_{\star}, \mu\right) & =M\left[\tau+\frac{g}{2} M-\frac{\epsilon}{6} \bar{\tau}\left(A-B \ln \left(\bar{\tau} / \mu^{2}\right)-\frac{\epsilon}{12}\left(\ln \left(\bar{\tau} / \mu^{2}\right)\right)^{2}\right)\right. \\
& \left.+\frac{\epsilon^{2}}{72} g M\left(1+I-2 \ln \left(\bar{\tau} / \mu^{2}\right)+\left(\ln \left(\bar{\tau} / \mu^{2}\right)\right)^{2}\right)\right]
\end{aligned}
$$

where

$$
\begin{aligned}
& A=1+\epsilon\left(\frac{13}{288}+\frac{53}{144} \ln \frac{4}{3}-\frac{1}{4} \ln 3-\frac{I}{3}\right) \\
& B=1+\epsilon\left(\frac{37}{288}+\frac{53}{144} \ln \frac{4}{3}\right) .
\end{aligned}
$$

An integral representation for $I=-2.3439072 \ldots$ is given in the appendix. In order to simplify the writing we will use hereafter the dimensionless quantities defined by

$$
\mu^{-2} \tau \rightarrow \tau \quad \mu^{-2} g M \rightarrow M \quad \mu^{-4} g q \rightarrow q .
$$

While equation (21) cannot be used directly to investigate the equation of state in the critical region $(\tau, M \rightarrow 0)$ one may map the critical region by an appropriate choice of the flow parameter $l$ in (19) on scales on which perturbation theory can be applied. For $l=M^{\nu / \beta}$ equations (21) and (19) yield the scaling form

$$
a q=M^{\delta} F\left(b \tau M^{-1 / \beta}\right)
$$

with the scaling variables $x=b \tau M^{-1 / \beta}$ and $z=a q M^{-\delta}$ and the universal scaling function

$$
\begin{aligned}
F(x)=x+1 & +\frac{\epsilon}{6} K[(x+2) \ln (x+2)-2(x+1) \ln 2] \\
& +\frac{\epsilon^{2}}{72}\left[(x+4)(\ln (x+2))^{2}-4(x+1)(\ln 2)^{2}\right]+\mathrm{O}\left(\epsilon^{3}\right)
\end{aligned}
$$

where

$$
K=1+\epsilon\left(\frac{85}{288}+\frac{29}{72} \ln 2-\frac{53}{144} \ln 3\right)=1+\epsilon 0.1699728 \ldots
$$


The coefficients $a$ and $b$ in equation (25) are

$$
\begin{gathered}
\left.a=2\left[1+\frac{\epsilon}{3}+\frac{\epsilon^{2}}{18}\left(\frac{85}{48}+\frac{53}{12} \ln 2-\frac{89}{24} \ln 3-\frac{5}{2} I\right)+\mathrm{O}\left(\epsilon^{3}\right)\right)\right] \\
b=2\left[1+\frac{1+\ln 2}{6} \epsilon+\frac{\epsilon^{2}}{36}\left(\frac{13}{48}-\frac{89}{24} \ln 3-3 I\right.\right. \\
\left.\left.+\left(\frac{99}{16}+\frac{59}{12} \ln 2-\frac{53}{24} \ln 3\right) \ln 2\right)+\mathrm{O}\left(\epsilon^{3}\right)\right] .
\end{gathered}
$$

They have been introduced in order to normalize the scaling function, i.e.,

$$
F(0)=1 \quad F(-1)=0 .
$$

The equation of state (25) satisfies the correct scaling behaviour in the critical region but the scaling function (26) can only be used if $x$ is not too large. Since $q$ is an analytic function of $M$ for positive $\tau$ (for equilibrium systems this was first discussed by Griffiths [34]) the approximation (26) breaks down for $M \ll \tau^{\beta}$. In order to study this limit one may choose the flow parameter in (19) as $l=\tau^{\nu}$. One arrives at

$$
a q=(b \tau)^{\beta \delta} \bar{F}\left(M(b \tau)^{-\beta}\right)
$$

with the scaling variables $y=M(b \tau)^{-\beta}=x^{-\beta}$ and $\bar{z}=a q(b \tau)^{-\beta \delta}=z y^{\delta}$ and the scaling function

$$
\begin{aligned}
\bar{F}(y)=y\{1+ & y+\frac{\epsilon}{6} K[(1+2 y) \ln (1+2 y)-2(1+y) \ln 2] \\
& \left.+\frac{\epsilon^{2}}{72}\left[(1+4 y)(\ln (1+2 y))^{2}-4(1+y)(\ln 2)^{2}\right]+\mathrm{O}\left(\epsilon^{3}\right)\right\}
\end{aligned}
$$

which is fully analytic in $y$ :

$$
\bar{F}(y)=y \sum_{k=0}^{\infty} c_{k} y^{k} .
$$

Of course $y^{-\delta} \bar{F}(y)$ and $F(x)$ match in the $\epsilon$-expansion up to second order.

\section{Parametric form of the equation of state}

The full range of the variables $\tau, M$ (including the active phase for $\tau<0$ ) can be investigated by introducing a parametric representation for the equation of state. For equilibrium systems such a representation was first suggested by Schofield et al. [26] and Josephson [27] and later derived in an $\epsilon$-expansion by Brézin et al. [28]. To apply this method to directed percolation we write $\tau$ and $M$ as

$$
b \tau=R(1-\theta) \quad M=R^{\beta} \theta / \theta_{0} .
$$

Inserting (34) with $\theta_{0}=2$ into (25) one finds

$$
a q=\left(R^{\beta} / 2\right)^{\delta} H(\theta)
$$


with the simple scaling function

$$
H(\theta)=\theta(2-\theta)+\mathrm{O}\left(\epsilon^{3}\right)
$$

The parameter range $R \geq 0,0 \leq \theta<2$ is required to describe the whole phase diagram around the critical point. The case $\tau<0, q \rightarrow+0$ corresponds to the limit $\theta \rightarrow 2$ (from below). (See figure 11.)

Figure 1. Sketch of the parametric representation (34). The straight lines correspond to a variation of $\theta$ at fixed $R$.

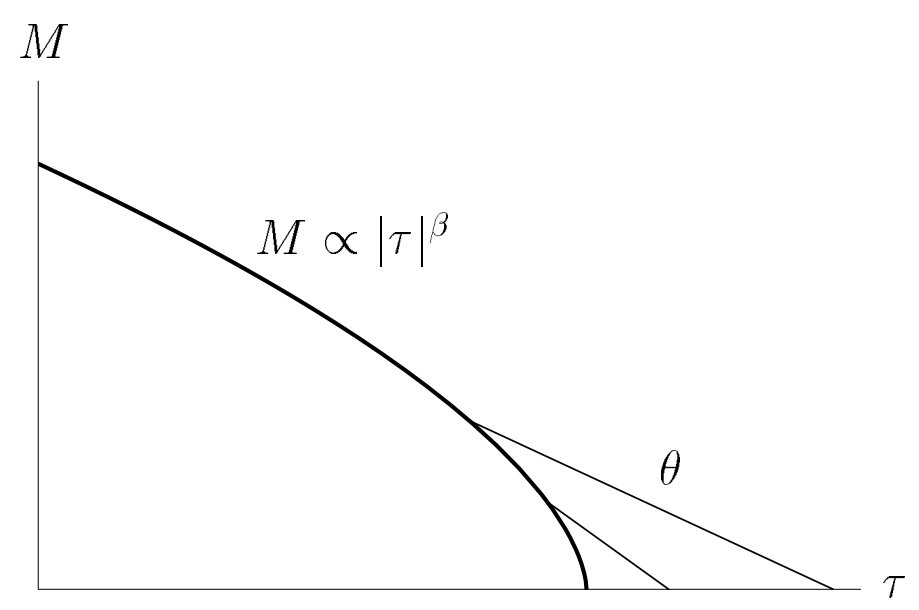

As a simple application of the parametric representation we briefly discuss the susceptibility

$$
\chi=\left.\frac{\partial M}{\partial q}\right|_{\tau}
$$

which satisfies a power law for $q \rightarrow 0$,

$$
\chi=\chi_{ \pm}|\tau|^{-\gamma}
$$

where $\chi_{+}$and $\chi_{-}$correspond to the cases $\tau>0$ and $\tau<0$, respectively, and $\gamma=\beta(\delta-1)$. To second order in $\epsilon$ the susceptibility can be written in terms of the parameters (34) as

$$
\chi=a 2^{\delta-1} R^{-\gamma} \frac{1-(1-\beta) \theta}{\beta \delta \theta(2-\theta)+2(1-\theta)^{2}} .
$$

Therefore the universal amplitude ratio $\chi_{-} / \chi_{+}$can be expressed to this order by the exponent $\beta$ as

$$
\begin{aligned}
\frac{\chi_{-}}{\chi_{+}} & =2 \beta-1+O\left(\epsilon^{3}\right)=1-\frac{\epsilon}{3}\left[1-\left(\frac{11}{288}-\frac{53}{144} \ln \frac{4}{3}\right) \epsilon+\mathrm{O}\left(\epsilon^{2}\right)\right] \\
& =1-\frac{\epsilon}{3}(1+\epsilon 0.0676885 \ldots) .
\end{aligned}
$$


The parametric equation of state allows us also to express the expansion coefficient $c_{0}$ in (33) to second order in $\epsilon$ by $\delta$. For this purpose we write

$x=2^{1 / \beta} \theta^{-1 / \beta}(1-\theta) \quad y=\frac{\theta / 2}{(1-\theta)^{\beta}} \quad z=\theta^{-\delta} H(\theta) \quad \bar{z}=\frac{2^{-\delta} H(\theta)}{(1-\theta)^{\beta \delta}}$

which gives to $\mathrm{O}\left(\epsilon^{2}\right)$

$$
\frac{\bar{F}(y)}{y}=\frac{\bar{z}}{y}=\frac{2^{1-\delta}(2-\theta)}{(1-\theta)^{\beta(\delta-1)}} .
$$

For $y \sim \theta \rightarrow 0$ this becomes

$$
\begin{gathered}
c_{0}=2^{2-\delta}+\mathrm{O}\left(\epsilon^{3}\right)=1-\frac{\epsilon}{3} \ln 2-\frac{\epsilon^{2}}{864}(85+164 \ln 2-106 \ln 3) \ln 2+\mathrm{O}\left(\epsilon^{3}\right) \\
=1-\epsilon 0.231049-\epsilon^{2} 0.0659639+\mathrm{O}\left(\epsilon^{3}\right) .
\end{gathered}
$$

\section{Conclusion}

We have derived a universal equation that describes the order parameter of directed percolation systems as a function of the scaling fields near the transition point to second order in $\epsilon=4-d$. Using a parametric representation for the thermodynamic variables our result could be written in a very simple form. This analysis was motivated by the success of a parametric equation of state [analogous to (34)-(36)] for equilibrium critical phenomena which was shown to be in good agreement with experiments [26]. Our hope is that our results will help to analyse data obtained by Monte Carlo simulations. It would be especially interesting to see how important the $O\left(\epsilon^{3}\right)$ corrections to Eq. (36) are in practice.

\section{Acknowledgment}

This work has been supported in part by the Sonderforschungsbereich 237 [Unordnung und Große Fluktuationen (Disorder and Large Fluctuations)] of the Deutsche Forschungsgemeinschaft.

\section{Appendix}

In this appendix we give some details of the diagrammatic analysis required to compute the equation of state. The diagrams contributing to two loop order are given in figure A1. The gaussian propagator follows from the action $\mathcal{J}_{G}$ in equation (8) as

$$
t \curvearrowright \stackrel{\mathbf{q}}{<}\left(t^{\prime}=\Theta\left(t-t^{\prime}\right) \exp \left(-\lambda\left(\bar{\tau}+q^{2}\right)\left(t-t^{\prime}\right)\right)\right.
$$


where $\Theta(t)$ denotes the step function and $\mathbf{q}$ is the momentum carried by the line. The correlator reads

$$
t \curvearrowright \mathbf{q} \leftrightharpoons t^{\prime}=\frac{g M}{2\left(\bar{\tau}+q^{2}\right)} \exp \left(-\lambda\left(\bar{\tau}+q^{2}\right)\left|t-t^{\prime}\right|\right)
$$

and the vertices

$$
\nwarrow=-\leftarrow<=\lambda g
$$

represent the interaction (9).

After integration over the internal time arguments the Feynman diagrams are

$$
\begin{aligned}
& \text { (a) }=-\frac{\lambda g^{2} M}{4} \int_{\mathbf{p}} \frac{1}{\bar{\tau}+p^{2}}=\frac{G_{\epsilon}}{\epsilon(2-\epsilon)} \lambda g^{2} M \bar{\tau}^{1-\epsilon / 2} \\
& \text { (b) }=\frac{\lambda g^{4} M}{12} \int_{\mathbf{k}, \mathbf{p}} \frac{1}{\left(\bar{\tau}+k^{2}\right)\left(\bar{\tau}+p^{2}\right)\left(\bar{\tau}+(\mathbf{k}+\mathbf{p})^{2}\right)}=\frac{\lambda g^{4} M}{12} \bar{\tau}^{1-\epsilon} I_{1} \\
& (\mathrm{c})=\frac{\lambda g^{4} M}{8} \int_{\mathbf{k}, \mathbf{p}} \frac{1}{\left(\bar{\tau}+k^{2}\right)^{2}\left(3 \bar{\tau}+k^{2}+p^{2}+(\mathbf{k}+\mathbf{p})^{2}\right)}=\frac{\lambda g^{4} M}{8} \bar{\tau}^{1-\epsilon} I_{2} \\
& (\mathrm{~d})=-\frac{\lambda g^{5} M^{2}}{16} \int_{\mathbf{k}, \mathbf{p}} \frac{1}{\left(\bar{\tau}+k^{2}\right)^{2}\left(\bar{\tau}+p^{2}\right)\left(\bar{\tau}+(\mathbf{k}+\mathbf{p})^{2}\right)} \\
& =\frac{\lambda g^{5} M^{2}}{16} \frac{1}{3} \frac{\partial}{\partial \bar{\tau}} \int_{\mathbf{k}, \mathbf{p}} \frac{1}{\left(\bar{\tau}+k^{2}\right)\left(\bar{\tau}+p^{2}\right)\left(\bar{\tau}+(\mathbf{k}+\mathbf{p})^{2}\right)}=\frac{\lambda g^{5} M^{2}}{16} \frac{1-\epsilon}{3} \bar{\tau}^{-\epsilon} I_{1}
\end{aligned}
$$

where we have used the notation $\int_{\mathbf{k}} \cdots=(2 \pi)^{-d} \int \mathrm{d}^{d} k \cdots$.

The $\epsilon$-expansions of the integrals $I_{1}$ and $I_{2}$ read

$$
\begin{aligned}
I_{1} & =\int_{\mathbf{k}, \mathbf{p}} \frac{1}{\left(1+k^{2}\right)\left(1+p^{2}\right)\left(1+(\mathbf{k}+\mathbf{p})^{2}\right)} \\
& =-\frac{6 G_{\epsilon}^{2}}{\epsilon^{2}}\left[1+\frac{3 \epsilon}{2}+\frac{\epsilon^{2}}{4}(7+I)+O\left(\epsilon^{3}\right)\right]
\end{aligned}
$$

with

$$
I=\int_{0}^{1} \mathrm{~d} x \frac{\ln (x(1-x))}{1-x(1-x)}=-2.3439072 \ldots
$$

and

$$
\begin{aligned}
I_{2} & =\int_{\mathbf{k}, \mathbf{p}} \frac{1}{\left(1+k^{2}\right)^{2}\left(3+k^{2}+p^{2}+(\mathbf{k}+\mathbf{p})^{2}\right)} \\
& =\frac{3 G_{\epsilon}^{2}}{2 \epsilon}\left[1+\frac{\epsilon}{2}(3-\ln 3)+O\left(\epsilon^{2}\right)\right] .
\end{aligned}
$$

The finite parts of the integrals are required to calculate the coefficient $K$ in the scaling function (26) and the normalization constants $a$ and $b(28,29)$. 
Figure A1. Contributions to the equation of state to two loop order.

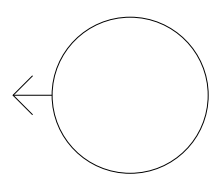

(a)

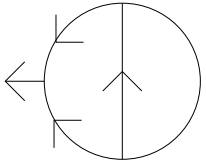

(b)

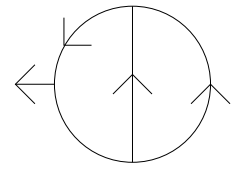

(c)

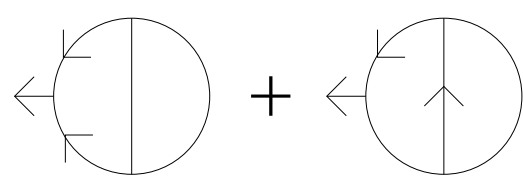

(d)

\section{References}

[1] Murray J D 1989 Mathematical Biology (Berlin: Springer)

[2] Dickman R and Burschka M A 1988 Phys. Lett. 127A 132-7

[3] Kertész J and Wolf D E 1989 Phys. Rev. Lett. 62 2571-4

[4] Schlögl F 1972 Z. Phys. 253 147-61

[5] Janssen H K 1981 Z. Phys. B 42 151-4

[6] Harris T E 1974 Ann. Probab. 2 969-88

[7] Liggett T M 1985 Interacting Particle Systems (New York: Springer)

[8] Kinzel W 1983 Percolation Structures and Processes ed G Deutsch, R Zallen and J Adler (Bristol: Hilger) pp 425-45

[9] Kinzel W 1985 Z. Phys. B 58 229-44

[10] Gribov V N 1968 Sov. Phys.-JETP 26 414-23

[11] Gribov V N and Migdal A A 1969 Sov. Phys.-JETP 28 784-95

[12] Moshe M 1978 Phys. Rep. 37C 255-345

[13] Grassberger P and Sundermeyer K 1978 Phys. Lett. 77B 220-2

[14] Grassberger P and de la Torre A 1979 Ann. Phys. (NY) 122 373-96

[15] Cardy J L and Sugar R L 1980 J. Phys. A: Math. Gen. 13 L423-7

[16] Obukhov S P 1980 Physica 101A 145-55

[17] Grassberger P 1982 Z. Phys. B 47 365-74

[18] Grassberger P and Zhang Y-C 1996 Physica A 224 169-79

[19] Jensen I 1996 J. Phys. A: Math. Gen. 29 7013-40

[20] Lauritsen K B, Sneppen K, Markošová M and Jensen M H 1997 Physica A 247 1-9

[21] Brézin E, Le Guillou J C and Zinn-Justin J 1976 Phase transitions and critical phenomena 6 ed C Domb and M S Green (London: Academic) pp 125-247

[22] Wallace D J 1976 Phase transitions and critical phenomena 6 ed C Domb and M S Green (London: Academic) pp 293-356

[23] Privman V, Hohenberg P C and Aharony A 1991 Phase transitions and critical phenomena 14 ed C Domb and J L Lebowitz (London: Academic)

[24] Kree R, Schaub B and Schmittmann B 1989 Phys. Rev. A 39 2214-21

[25] van Wijland F, Oerding K and Hilhorst H J 1998 Physica A 251 179-201

[26] Schofield P, Litster J D and Ho J T 1969 Phys. Rev. Lett. 23 1098-102

[27] Josephson B D 1969 J. Phys. C: Solid State Phys. 2 1113-5

[28] Brézin E, Wallace D J and Wilson K G 1972 Phys. Rev. Lett. 29 591-4

[29] Janssen H K 1976 Z. Phys. B 23 377-80 
[30] De Dominicis C 1976 J. Physique (Paris) C 37 247-53

[31] Bausch R, Janssen H K and Wagner H 1976 Z. Phys. B 24 113-27

[32] Janssen H K 1992 From Phase Transitions to Chaos, Topics in Modern Statistical Physics ed G Györgyi, I Kondor, L Sasvári and T Tél (World Scientific, Singapore) pp 68-91

[33] Martin P C, Siggia E D and Rose H A 1973 Phys. Rev. A 8 423-37

[34] Griffiths R B 1967 Phys. Rev. 158 176-87 